\title{
Development of Severe Hypophosphatemia from Acquired Fanconi Syndrome during Treatment with Abiraterone
}

\author{
Chamberlain $\mathrm{ED}^{1}$, Vanek $\mathrm{C}^{2}$, Alumkal JJ ${ }^{3}$ and Graff JN $\mathrm{N}^{* 3,4}$
}

${ }^{1}$ Department of Medicine, Oregon Health \& Science University, Portland, OR

${ }^{2}$ Division of Endocrinology, Oregon Health \& Science University, Portland, OR

${ }^{3}$ Knight Cancer Institute, Oregon Health \& Science University, Portland, OR

${ }^{4}$ Portland VA Medical Center, Portland, OR

*Corresponding author: Graff JN, 3710 SW US Veterans Hospital Road, MC P3CHEMO, Portland, OR 97239, Tel: 503-220-8262 x 55688, Fax: 503-494-6197, E-mail: graff@ohsu.edu

Citation: Chamberlain ED, Vanek C, Alumkal JJ, Graff JN (2015) Development of Severe Hypophosphatemia from Acquired Fanconi Syndrome during Treatment with Abiraterone. J Case Rep Stud 3(1): 101. doi: $10.15744 / 2348-9820.2 .401$

Received Date: November 12, 2014 Accepted Date: January 30, 2015 Published Date: February 03, 2015

\section{Abstract}

Objectives: To report two cases of patients with metastatic, castration resistant prostate cancer who developed profound hypophosphatemia while on treatment with abiraterone.

Case Summary: The first patient was a 77 year old man who had been on abiraterone for 12 months before his phosphorous was checked. At that point, it was $0.6 \mathrm{mg} / \mathrm{dl}$, which is severely decreased. A full work up for hypophosphatemia showed normal parathyroid hormone and 25-hydroxyvitamin D. A urine study showed inappropriate excretion of phosphorous along with aminoaciduria and normoglycemic glucosuria. The phosphorous level slowly improved upon discontinuation of abiraterone and aggressive repletion of his phosphorous with elemental phosphorous and calcitriol. The second patient was a 74 year old man who had a slightly decreased phosphorous prior to abiraterone initiation. It steadily fell during abiraterone therapy down to $0.9 \mathrm{mg} / \mathrm{dl}$. Aggressive repletion would not raise the phosphorous to normal. It only improved upon discontinuation of abiraterone. The results of the work up were similar to those of the first patient.

Discussion: These two patients had profound hypophosphatemia. Phosphorous levels are not routinely checked by clinicians, and the severe hypophosphatemia in these patients demonstrates how important it is to check this level. We propose that the mechanism of phosphorous loss is through inappropriate renal wasting by Fanconi syndrome.

Conclusion: Hypophosphatemia occurs in prostate cancer patients on abiraterone. It should be monitored closely in patients on this therapy.

\section{Introduction}

Abiraterone acetate $\left(Z_{y t i g a}{ }^{\infty}\right)$ inhibits androgen biosynthesis by acting on the enzyme CYP17 [1]. In two phase III clinical trials, abiraterone plus prednisone compared to prednisone alone improved survival in men with metastatic, castration-resistant prostate cancer (mCRPC) [2,3]. Abiraterone initially received Food and Drug Administration approval in 2011 for treatment of patients who had received chemotherapy [4], and the approval was expanded in 2012 to include those who are chemotherapynaïve [5]. In the phase III clinical trials, the common adverse effects observed in the abiraterone arms were often related to mineralocorticoid excess or glucocorticoid deficiency as predicted by its role in blocking a critical step of steroidogenesis. However, hypophosphatemia was also commonly reported, and this is not explained by inhibition of CYP17. Here, we report two cases of patients with mCRPC treated with abiraterone who were found to have profound hypophosphatemia. We propose the mechanism for their hypophosphatemia as inappropriate renal excretion of phosphate due to Fanconi syndrome acquired from treatment with abiraterone.

Common symptoms of severe hypophosphatemia, defined as serum phosphate $<1.0 \mathrm{mg} / \mathrm{dl}$ (or $<0.32 \mathrm{mmol} / \mathrm{L}$ ), are muscle weakness and bone pain due to phosphate's critical role in both muscle function via adenosine triphosphate (ATP) and bone composition in the form of crystalline hydroxyapatite $\left[\mathrm{Ca} 10\left(\mathrm{PO}_{4}\right) 6(\mathrm{OH})_{2}\right][6]$. Severe hypophosphatemia can be life threatening since phosphate is vital to cardiac and respiratory function. There are three primary mechanisms of hypophosphatemia: increased renal excretion, decreased intestinal absorption, and transcellular shift (extracellular to intracellular) [7]. The first mechanism is due to dysfunctional proximal tubular reabsorption of the phosphate anion. This decrease in proximal renal tubular resorption of phosphate is part of the disease entity called Fanconi syndrome and results in urinary loss of phosphate, glucose, amino acids, and other solutes.

Certain medications are known to cause acquired Fanconi syndrome, including agents from antibacterial, antiviral, antiretroviral, anticonvulsant, and anticancer drug classes [8]. Here we present two patients who developed acquired Fanconi syndrome during treatment with abiraterone. These cases, to our knowledge, mark the first documentation of this phenomenon. 


\section{Case 1}

A 77 year-old man presented with an initial diagnosis of metastatic prostate cancer to the bones in 2009. Medical castration with the luteinizing hormone releasing hormone (LHRH) agonist, leuprolide acetate, led to an initial decrease in prostate-specific antigen (PSA). Unfortunately, his disease progressed over the ensuing twelve months prompting initiation of concomitant antiandrogen therapy with bicalutamide followed by systemic therapy with docetaxel chemotherapy, which was discontinued after one cycle for significant anorexia and bleeding. His mCRPC was subsequently treated in September of 2011 with abiraterone (1000 mg orally daily) in combination with prednisone $5 \mathrm{mg}$ orally twice a day. He began the intravenous bisphosphonate zoledronic acid in November 2011 for his bone metastases. His cancer responded well to abiraterone, demonstrated by an interval decrease in PSA from $540 \mathrm{ng} / \mathrm{ml}$ (September 2011) to $19 \mathrm{ng} / \mathrm{ml}$ (January 2012). However, this response was not durable, marked by a rise in PSA to $111 \mathrm{ng} / \mathrm{ml}$ (July 2012).

While considering clinical trial participation, routine lab work demonstrated a critically low serum phosphorous of $0.6 \mathrm{mg} / \mathrm{dl}$ (range 2.5-4.5 mg/dl). This abnormality was discovered during ongoing treatment with abiraterone (September 2012), a full year after initiation. Neither his referring oncologist nor primary care provider had checked the phosphorous either prior to or during treatment with abiraterone. Endocrinology evaluated the patient for his critical hypophosphatemia. The patient reported persistent chronic knee pain but was otherwise asymptomatic. The differential included vitamin D deficiency, gastrointestinal malabsorption, oncogenic osteomalacia, exposure to zoledronic acid, and renal phosphorous wasting from hyperparathyroidism or a renal tubule defect from an acquired Fanconi syndrome. The patient had normal vitamin D (25-OH) levels, lack of clinical findings suggestive of malabsorption, normal PTH, a new findings of high urine glucose of $500 \mathrm{mg} / \mathrm{dl}$ (normal range 0-15) in the setting of normal non-fasting serum glucose of $113 \mathrm{mg} / \mathrm{dl}$ (normal <140) and $2+$ glycine on qualitative amino acid urine screen (Table 1$)$. A normal SPEP ruled out light chain deposition disease as a cause for acquired Fanconi syndrome. Comparison of phosphate and creatinine in the serum and urine revealed a decreased ratio of renal tubular maximum reabsoroption rate of phosphate to the glomerular filtration rate (TmP/GFR) [3]. This established inappropriate urinary loss of phosphate due to a proximal renal tubule defect. This, together with his normoglycemic glucosuria and aminoaciduria, highly suggests the diagnosis of acquired Fanconi syndrome. Of note, his medication list lacked drugs well-known to cause acquired Fanconi syndrome.

\begin{tabular}{|c|c|c|c|}
\hline Lab & Patient 1 & Patient 2 & Range, Units \\
\hline Serum phosphorous & 0.6 & 0.9 & $2.4-4.7, \mathrm{mg} / \mathrm{dl}$ \\
\hline Urine phosphorous & 54 & 52.3 & No range, $\mathrm{mg} / \mathrm{dl}$ \\
\hline Serum creatinine & 0.89 & 0.6 & $0.70-1.30, \mathrm{mg} / \mathrm{dl}$ \\
\hline Urine creatinine & 83.9 & 82 & No range, $\mathrm{mg} / \mathrm{dl}$ \\
\hline $\begin{array}{l}\text { Fractional excretion of } \\
\text { phosphorous }\end{array}$ & 95 & 42 & $\begin{array}{l}\text { Normal }<15 \% \text { if } \\
\text { hypophosphatemia }\end{array}$ \\
\hline Non-fasting Serum glucose & 113 & 97 & $<200, \mathrm{mg} / \mathrm{dl}$ \\
\hline Urine glucose & 500 & 24 & $0-15, \mathrm{mg} / \mathrm{dl}$ \\
\hline Urine amino acids & $\begin{array}{l}2+(\text { qualitative }) \\
(\text { normal }=0)\end{array}$ & $\begin{array}{l}\text { Alanine } 73(9-67) \\
\text { Proline } 4(<3) \\
\text { Tyrosine } 23(3-19) \\
\text { Valine } 7(2-5) \\
\text { Phenylalanine } 18(2-9) \\
\text { Tryptophan } 21(2-14)\end{array}$ & No range, $\mathrm{mmol} / \mathrm{mol} \mathrm{Cr}$ \\
\hline 25-hydroxy Vitamin D & 28 & 41 & $20-80, \mathrm{ng} / \mathrm{ml}$ \\
\hline PTH & 56 & 76 & $15-65, \mathrm{pg} / \mathrm{ml}$ \\
\hline Calcium & 8.1 & 8.6 & $8.6-10.2, \mathrm{mg} / \mathrm{dl}$ \\
\hline Albumin & 3.8 & 3.7 & $3.5-4.7, \mathrm{~g} / \mathrm{dl}$ \\
\hline SPEP & Normal & Normal & No paraprotein \\
\hline
\end{tabular}

Table 1: Selected Lab Values

He discontinued abiraterone treatment and initiated potassium-phosphate supplementation as well as calcitriol to aid intestinal phosphorous absorption. His zoledronic acid was also discontinued out of concern it could be exacerbating the hypophosphatemia. Repeat lab work demonstrated an improved serum phosphorus level of $1.6 \mathrm{mg} / \mathrm{dl}$, though persistent glucosuria. Six months after the diagnosis of Fanconi syndrome, he declined further follow-up for both his acquired Fanconi syndrome and metastatic prostate cancer.

\section{Case 2}

A 74 year-old man was diagnosed with Gleason $4+3$ adenocarcinoma of the prostate and was treated with external beam radiation therapy in 2008. In 2012, he was found to have metastatic cancer to the bones and began treatment with leuprolide and bicalutamide. He also started denosumab for his bone metastases, which he received for 12 months. His cancer progressed, and in October 2013, he started abiraterone (1000 mg orally daily) with prednisone $5 \mathrm{mg}$ orally twice a day. At the same time, his denosumab was changed to zoledronic acid when he changed medical systems. 
When he began abiraterone acetate with prednisone, he had a baseline phosphorous of $2.2 \mathrm{mg} / \mathrm{dl}$. Within one month of beginning abiraterone and prednisone, his phosphorous fell to $1.6 \mathrm{mg} / \mathrm{dl}$ and $0.9 \mathrm{mg} / \mathrm{dl}$ at four months. Work up of his hypophosphatemia revealed a normal 25-hydroxy vitamin D level, lack of clinical findings for malabsorption and a mild elevation in PTH (76 pg/ml). His serum and urine studies revealed a decreased TmP/GFR consistent with inappropriate renal excretion of phosphorus (Table 1). Aminoaciduria and normoglycemic glucosuria were also present and are not features of hyperparathyroidism. The patient was diagnosed with abiratrone induced acquired Fanconi syndrome.

He continued on abiraterone because he had continued clinical response. Phosphorus supplementation was initiated in the form of sodium phosphorous/potassium phosphorous (Neutra-Phos) $250 \mathrm{mg}$ twice a day. However, his severe hypophosphatemia required oral doses of $1500 \mathrm{mg}$ per day, plus potassium phosphorous $500 \mathrm{mg}$ twice a week intravenously. He was hospitalized for a cardiac event and received potassium phosphorous $1500 \mathrm{mg}$ per day intravenously for six consecutive days. This raised his serum phosphorous to $2.0 \mathrm{mg} / \mathrm{dl}$. He also began to take calcitriol $0.25 \mathrm{mg}$ orally per day. Despite the efforts to raise his phosphorous, his risk of profound hypophosphatemia leading to life-threatening cardiac dysrhythmias in setting of his underlying cardiac disease that included coronary artery disease status post bypass grafting was felt to outweigh the benefit of abiratrone. Abiraterone was discontinued. Four weeks later, in the setting of continued oral and intravenous supplemental phosphorous repletion, calcitriol and zoledronic acid, his phosphorous rose to $1.9 \mathrm{mg} / \mathrm{dl}$.

\section{Discussion}

Here we report two cases of hypophosphatemia resulting from acquired Fanconi syndrome during treatment with abiraterone acetate for mCRPC. Each patient demonstrated classical features of Fanconi syndrome: a disease of renal dysfunction of reduced proximal tubule reabsorption threshold causing hypophosphatemia, normoglycemic glucosuria, and aminoaciduria.

Fanconi syndrome is associated with a wide variety of both inherited and acquired conditions. This disease was first described in 1924 and better defined in 1936 by Guido Fanconi, for whom the disease is named, who evaluated children presenting with rickets, growth retardation, and glucosuria. Although the underlying pathology has not been clearly elucidated, experimental models using a compound, maleic acid, showed renal tubule dysfunction characterized by defective luminal entry of the compound, excessive back leak, or interference with $\mathrm{Na}, \mathrm{K}$, -ATPase activity [9]. Known drug offenders leading to acquired Fanconi syndrome include drugs from antibacterial [10], antiviral [11], antiretroviral [12,13] and anticonvulsant classes [14]. Known associations within the anticancer drug classes include ifosfamide, cisplatin, carboplatin, streptozocin, azacitidine, suramin, and mercaptopurine [5]. Abiraterone acetate has yet to be included on this list.

These patients were on zoledronic acid, and this agent was linked to Fanconi syndrome in two previously published case reports $[15,16]$. Another key differential etiology to consider includes the rare paraneoplastic syndrome called oncogenic osteomalacia, characterized by renal phosphate wasting and severe hypophosphatemia leading to osteomalacia. It is most often seen with mesenchymal tumors, though a literature review spanning thirty-four years (1966-2000) revealed 24 case reports of this syndrome in patients with metastatic prostate cancer [17]. Two more cases have been reported since $[18,19]$. Unfortunately, we do not have a baseline serum phosphorous level for the first patient, and he declined follow-up after discontinuing abiraterone. While we do not know exactly when the hypophosphatemia developed in the first patient, it could have developed long before it was discovered. Either bisphosphate therapy or a paraneoplastic syndrome leading to hypophosphatemia is possible. However, the second patient had a near normal baseline serum phosphorous before treatment despite having widely metastatic disease which makes a paraneoplastic syndrome less likely. He developed severe hypophosphatemia while taking both abiraterone and zoledronic acid. However, his phosphorous began to resolve after stopping the abiraterone despite continued therapy with zoledronic acid. This supports the argument that abiraterone alone led to this patient's hypophosphatemia.

In the two phase III clinical trials of abiraterone with prednisone versus placebo with prednisone, hypophosphatemia was seen more commonly in the abiraterone treated groups [2,3]. Any grade of hypophosphatemia occurred in $23.8 \%$ on the abiraterone arm versus $15.7 \%$ on the placebo arm [5]. Significant hypophosphatemia (serum phosphorous $<2 \mathrm{mg} / \mathrm{dl}$ ) was seen in $7.2 \%$ on abiraterone versus $5.8 \%$ on placebo. There are no reports from these studies of aminoaciduria or glycosuria in the setting of normal serum glucose. Although the differential for hypophosphatemia is broad and includes vitamin D deficiency, nutritional deficiency, hyperparathyroidism, oncogenic osteomalacia, or concurrent bisphosphonate therapy, it is unclear what caused it in the clinical trials, though we retrospectively propose abiraterone induced acquired Fanconi syndrome as a potential mechanism for this observed abnormality.

Drug induced Fanconi syndrome is usually reversible by discontinuing the offending agent and initiating vitamin and mineral supplementation to make up for urinary losses. However, despite initiating these interventions, permanent renal damage may occur and long term sequelae may be severe with the spectrum of renal disease ranging from subclinical and biochemical evidence of renal damage to irreversible damage and bone disease (osteomalacia) from urinary excretion of phosphate. Prognosis of druginduced Fanconi syndrome varies according to the offending medication. Reversal of Fanconi syndrome caused by outdated tetracyclines, for example, may take up to one year [20]. Whereas valproic acid induced Fanconi syndrome usually improves promptly with discontinuation of the medication [14]. 
We suggest that clinicians regularly monitor serum phosphorus and obtain urine analyses for detection of glucosuria or proteinuria. If proteinuria is found, the urinanalysis should include either quantitative or qualitative amino acid analysis. Upon making the diagnosis of drug induced Fanconi syndrome, the clinician should consider discontinuation of abiraterone and administer phosphorous and calcitriol supplementation, which aids in phosphorous absorption, and continue serial lab and urine monitoring.

We can expect continued wide use of abiraterone acetate for patients with mCRPC, and it is clear that careful monitoring of electrolytes such as potassium and liver function tests is necessary. Our case reports demonstrate that hypophosphatemia due to acquired Fanconi syndrome is another important side effect of treatment with abiraterone. We propose that patients treated with this medication undergo regular laboratory testing including serum phosphorus levels. By regularly monitoring patients for early signs of disease with these simple laboratory evaluations, Fanconi syndrome can be detected early and potential irreversible damage may be prevented.

\section{References}

1. Attard G, Reid AH, Auchus RJ, Hughes BA, Cassidy AM, et al. (2012) Clinical and biochemical consequences of CYP17A1 inhibition with abiraterone given with and without exogenous glucocorticoids in castrate men with advanced prostate cancer. J Clin Endocrinol Metab 97: 507-16.

2. de Bono JS, Logothetis CJ, Molina A, Fizazi K, North S, et al. (2011) Abiraterone and increased survival in metastatic prostate cancer. N Engl J Med 364: 19952005.

3. Ryan CJ, Smith MR, de Bono JS, Molina A, Logothetis CJ, et al. (2013) Abiraterone in metastatic prostate cancer without previous chemotherapy. N Engl J Med 368: $138-48$.

4. Package Insert. Zytiga (abiraterone acetate) oral tablets. Horsham, PA: Centocor Ortho Biotech Inc, 2011.

5. Product Information. Zytiga (abiraterone acetate) oral tablets. Horsham, PA: Centocor Ortho Biotech Inc, 2011.

6. Gaasbeek A, Meinders AE (2005) Hypophosphatemia: an update on its etiology and treatment. Am J Med 118: 1094-101.

7. Imel EA, Econs MJ (2012) Approach to the hypophosphatemic patient. J Clin Endocrinol Metab 97: 696-706.

8. Izzedine H, Launay-Vacher V, Isnard-Bagnis C, Deray G (2003) Drug-induced Fanconi’s syndrome. Am J Kidney Dis 41: 292-309.

9. Bergeron M, Dubord L, Hausser C, Schwab C (1976) Membrane permeability as a cause of transport defects in experimental Fanconi syndrome. A new hypothesis. J Clin Invest 57: 1181-9.

10. Melnick JZ, Baum M, Thompson JR (1994) Aminoglycoside-induced Fanconi’s syndrome. Am J Kidney Dis 23:118-22.

11. Jung YK, Yeon JE, Choi JH, Kim CH, Jung ES, et al (2010) Fanconiss Syndrome Associated with Prolonged Adefovir Dipivoxil Therapy in a Hepatitis B Virus Patient. Gut Liver 4: 389-93.

12. Izzedine H, Harris M, Perazella MA (2009) The nephrotoxic effects of HAART. Nat Rev Nephrol 5: 563-73.

13. Morris AA, Baudouin SV, Snow MH (2001) Renal tubular acidosis and hypophosphataemia after treatment with nucleoside reverse transcriptase inhibitors. AIDS 15: 140-1.

14. Watanabe T, Yoshikawa H, Yamazaki S, Abe Y, Abe T (2005) Secondary renal Fanconi syndrome caused by valproate therapy. Pediatr Nephrol 20: 814-7.

15. Torimoto K, Okada Y, Arao T, Mori H, Tanaka Y (2012) A case of zoledronate-induced tubulointerstitial nephritis with Fanconi syndrome. Endocrine J 59: 1051-6.

16. Yoshinami T, Yagi T, Sakai D, Sugimoto N, Imamura F (2011) A case of acquired Fanconi syndrome induced by zoledronic acid. Intern Med 50: 1075-9.

17. Pelger RC, Lycklama A Nijeholt GA, Papapoulos SE, Hamdy NA (2005) Severe hypophosphatemic osteomalacia in hormone-refractory prostate cancer metastatic to the skeleton: natural history and pitfalls in management. Bone 36: 1-5.

18. Chiam P, Tan HC, Bee YM, Chandran M (2013) Oncogenic osteomalacia -- hypophosphataemic spectrum from "benignancy" to "malignancy". Bone 53: 182-7.

19. Mak MP, da Costa e Silva VT, Martin RM, Lerario AM, Yu L, et al. (2012) Advanced prostate cancer as a cause of oncogenic osteomalacia: an underdiagnosed condition. Support Care Cancer 20: 2195-7.

20. Fulop M, Drapkin A (1965) Potassium-Depletion Syndrome Secondary to Nephropathy Apparently Caused by "Outdated Tetracycline". N Engl J Med 272: 986-9.

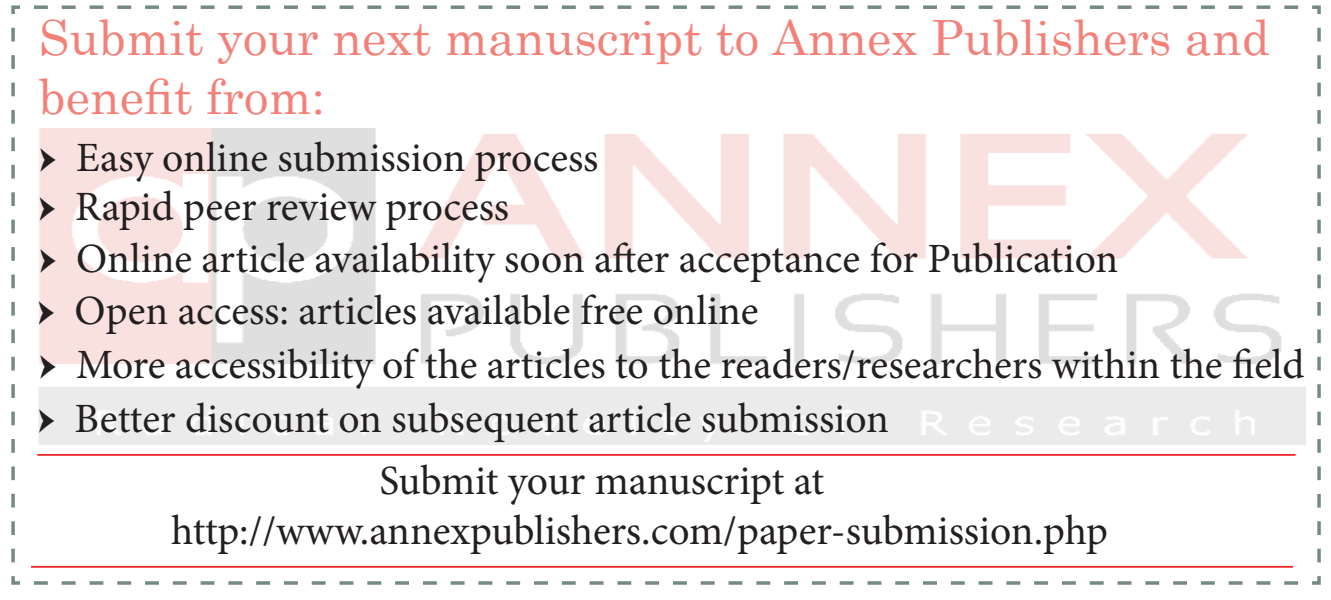

